\title{
New and Emerging Treatments for Lymphedema
}

\author{
Mark V. Schaverien, MBChB, MD, MSc, MEd, FRCS (Plast) ${ }^{1}$ Melissa B. Aldrich, PhD $^{2}$ \\ ${ }^{1}$ Department of Plastic Surgery, University of Texas MD Anderson \\ Cancer Center, Houston, Texas \\ 2 Center for Molecular Imaging, Brown Institute for Molecular \\ Medicine, UT Health, Houston, Texas \\ Address for correspondence Mark V. Schaverien, MBChB, MD, MSc, \\ MEd, FRCS (Plast), Department of Plastic Surgery, University of Texas \\ MD Anderson Cancer Center, Unit 1488, 1400 Pressler Street, \\ Houston, TX 77030 (e-mail: mvschaverien@mdanderson.org).
}

Semin Plast Surg 2018;32:48-52.

\begin{abstract}
Keywords

- lymphedema

- treatment

- therapies

- inflammation

Although nonoperative and operative treatments for lymphedema (LE) are well established, these procedures typically provide only partial relief from limb swelling, functional impairment, and the risk of cellulitis. The lack of a cure for $L E$, however, is due to an incomplete understanding of the underlying pathophysiological mechanisms, and current research efforts are focusing on elucidating these processes to provide new, targeted therapies for this prevalent disease for which there is no cure. This article reviews the current literature regarding the pathophysiological mechanisms that underlie LE, as well as new and emerging therapies for the condition.
\end{abstract}

The lymphatic system plays a crucial role in fluid homeostasis, immunity, and lipid reabsorption. When lymphatic injury occurs as a result of lymphadenectomy, especially when followed by radiation therapy to the surgical field, ${ }^{1}$ lymphatic fluid flow is obstructed, leading to lymphatic vessel distention, valve dysfunction, and reflux into the surrounding soft tissues. This results in a chronic inflammatory process involving the dermis and subcutaneous tissues, as well as differentiation of fibroblasts into myofibroblasts, leading to increased collagen deposition and remodeling of the extracellular matrix. ${ }^{2}$ The development of collateral lymphatic vessels may be inadequate to remove the lymphatic fluid accumulation in the interstitial space, leading to stasis, setting in motion a vicious cycle toward chronic lymphedema (LE), resulting in further inflammation, impaired lymphatic function, progressive fibrosis, and sclerosis, with eventual obliteration of the lymphatic vessel lumen and adipose tissue differentiation. ${ }^{2-7}$

The absence of LE in some patients despite lymphatic injury and the delayed development of the disease in others indicate that genetic and physiological factors interplay with secondary events to drive the progression from a subclinical LE steady state into the clinical LE phenotype. This article reviews the current evidence regarding the pathophysiology of LE, as well as new and emerging treatment modalities.

\section{Inflammatory Mechanisms in LE}

Lymphatic fluid stasis following lymphatic injury leads to infiltration of a diverse range of inflammatory cells, including lymphocytes, macrophages, neutrophils, and dendritic cells. $^{8}$ Multiple mechanisms are involved in this chronic inflammatory process, providing several potential targets for prevention and treatment of LE.

\section{CD4+ Cell T Helper 2 (Th2) Subtype}

Current evidence supports the key role of T cells, in particular $\mathrm{CD}^{+}$cells, in mediating the inflammatory process seen in $\mathrm{LE}^{7,9-11}$ with positive linear correlation between the number of $\mathrm{CD}^{+}$cells and the severity of disease in lymphedematous tissues, as indicated by lymphatic vessel remodeling and collecting vessel impairment. ${ }^{5,12}$ In the absence of $\mathrm{CD} 4^{+}$ cells or where $\mathrm{CD}^{+}$cells are specifically neutralized by monoclonal antibodies, LE does not develop. ${ }^{5}$ Interestingly, lymphatic injury through lymphadenectomy leads to activation and release of $\mathrm{CD}^{+}$cells from regional lymph nodes with increased expression of skin-homing receptors, accompanied by an increase in the relevant ligands in lymphedematous skin. ${ }^{8}$ Tacrolimus, which inhibits $\mathrm{T}$ cell proliferation and differentiation when applied topically, prevents the development of LE, ${ }^{13}$ and there are plans for a future clinical trial.
Issue Theme Lymphedema Management; Guest Editors: Edward I. Chang, MD, FACS, Mark V. Schaverien, $M B C h B, M D, M S c, M E d$, FRCS (Plast), and Jesse C. Selber, MD, MPH, FACS
Copyright $\odot 2018$ by Thieme Medical Publishers, Inc., 333 Seventh Avenue, New York, NY 10001, USA. Tel: +1(212) 584-4662.
DOI https://doi.org/ 10.1055/s-0038-1632403. ISSN 1535-2188. 
Specifically, the $\mathrm{CD}^{+}$cell $\mathrm{T}$ helper 2 (Th2) subtype is essential for the development of lymphatic dysfunction and fibrosis in mice and humans, ${ }^{14}$ and lymphatic injury results in a Th2-biased response with infiltration of large numbers of $\mathrm{CD}^{+}$ interleukin- $4^{+}{\left.\text {(IL- } 4^{+}\right)}$IL-13 ${ }^{+}$Th2 cells. ${ }^{5,14}$ Th2 cytokines, including IL-4, IL-13, interferon-gamma (IFN- $\gamma$ ), and transforming growth factor $\beta-1$ (TGF- $\beta 1$ ), inhibit collateral lymphatic vessel formation by downregulating lymphatic endothelial cell (LEC) genes, resulting in inhibition of LEC survival, proliferation, migration, and tubule migration, as well as mediation of fibrosis by differentiation of fibroblasts to myofibroblasts. ${ }^{11,14,15}$ Monoclonal antibodies to IL-4 or IL-13 can block Th2 differentiation to prevent the development of soft tissue fibrosis and lymphatic dysfunction resulting from LE. ${ }^{14-16}$

Collectively, these results suggest that $\mathrm{CD} 4^{+} \mathrm{T}$ cells, more specifically Th2 helper cells, are required for the development of LE and that the Th2 cytokines IL-4 and IL-13 are antilymphangiogenic, contributing to the pathophysiology of LE. Fibrosis as mediated by Th2 cytokines is critical to the pathology of LE, likely due to the progressive obliteration of lymphatic vessel lumen, as well as impairing collateral lymphatic growth. A clinical trial is currently underway evaluating the efficacy of a combination of IL- 4 and IL-13 monoclonal antibodies in the treatment of early breast cancer-related upper extremity LE (BCRL).

\section{T Regulatory Cells}

$T$ regulatory cells (Tregs) are downregulated in both murine models of LE and in humans with LE. ${ }^{5}$ Depletion of Tregs leads to exacerbation of edema, concomitant with increased infiltration of immune cells; conversely, expansion of Tregs significantly reduces LE development. ${ }^{17}$ These findings suggest that that Treg application may constitute a potential future treatment modality for LE. ${ }^{18}$

\section{Transforming Growth Factor $\boldsymbol{\beta}-1$}

Findings in murine models of LE as well as in clinical biopsy specimens have suggested that TGF- $\beta 1$ plays a key role in the regulation of fibrosis and lymphatic dysfunction in LE. 7,19,20 Inhibition of TGF- $\beta$ results in decreased fibrosis and improved lymphatic vessel function, suggesting that this cytokine plays an essential role in the development of fibrosis. ${ }^{7,19}$

\section{Interleukin-6}

Chronic LE, in addition to fibrosis, is characterized by adipose tissue differentiation. Lymphatic fluid stasis can initiate adipose tissue differentiation. ${ }^{21}$ IL-6, a known regulator of adipose tissue homeostasis, is increased in patients with LE, and in murine models of LE, IL-6 appears to be an inhibitor of adipose tissue deposition and may function to limit the pathologic effects of LE. ${ }^{22}$ IL-6 may therefore be a potential target for future treatment to prevent adipose tissue differentiation in chronic LE states and to possibly prevent its recurrence following debulking surgeries.

\section{Induced Nitric Oxide Synthase}

Lymphatic vessel peristalsis is mediated by perilymphatic smooth muscle cell contraction, and this intrinsic lymphatic pumping mechanism is regulated by gradients of nitric oxide (NO) produced by LEC-derived nitric oxide synthase, leading to coordinated cell contraction and relaxation. ${ }^{23,24}$ Patients with LE have decreased lymphatic pumping pressures. ${ }^{25}$ The perilymphatic inflammatory cell infiltrate that characterizes LE upregulates the expression of induced nitric oxide synthase (iNOS), disrupting the intrinsic NO gradient, thereby decreasing collecting lymphatic contractility and lymphatic fluid transport. iNOS causes a reduction in the strength of lymphatic contraction through excessive relaxation of collecting lymphatic vessels and inhibition of iNOS-producing cells significantly restores lymphatic contraction. ${ }^{26}$ iNOS-induced impairment in lymphatic transport not only leads to the accumulation of lymphatic fluid, but also contributes to immunosuppression by reducing antigen transport to the regional lymph nodes. ${ }^{24}$

\section{Macrophages}

Although $\mathrm{CD}^{+}$cells have a central role in the pathophysiology of LE, they are not the only cells involved, and the role of macrophages in acute LE is also key. ${ }^{27,28}$ In the acute phase of LE, Th1 and Th17 cells play an important role through macrophage activation, subsequent vascular endothelial growth factor-C (VEGF-C) secretion, and finally, lymphangiogenesis. $\mathrm{CD}^{+} \mathrm{T}$ cells interact with macrophages, leading to VEGF-C expression, which then promotes the development of immature lymphatic vessels in initial LE. ${ }^{29} \mathrm{M} 2$ macrophages are therefore likely key regulators of the regeneration of collateral lymphatics after lymphatic injury. ${ }^{20}$ Macrophage depletion after the establishment of LE, however, results in decreased lymphatic transport activity and VEGF-C expression, as well as enhanced tissue fibrosis response, possibly secondary to the increased $\mathrm{CD}^{+}$cell accumulation. ${ }^{28,30}$ In addition, macrophages promote the expression of iNOS. These studies suggest that macrophages play a complex role in the pathophysiology of LE by directly and indirectly regulating the tissue fibrosis response, lymphangiogenesis, and lymphatic vessel pumping.

\section{Leukotriene B4}

Leukotriene B4 (LTB4) levels are significantly elevated in human postsurgical LE patients. ${ }^{31}$ LTB4 in LE is produced by macrophages. ${ }^{32}$ Favorable results were observed in a murine LE model using ketoprofen ${ }^{33}$ to specifically antagonize LTB4, reversing LE and improving lymphatic vascular function, as well as diminishing macrophage, neutrophil, and $\mathrm{CD}^{+} \mathrm{T}$ cell infiltration into the lymphedematous tissue. ${ }^{31,34}$ LTB4 antagonism may therefore represent a promising approach for the treatment of LE, and a phase 2 randomized, double-blind, placebo-controlled multicenter study is currently underway to investigate the effectiveness of an inhibitor of leukotriene A4 hydrolase (LTA4H), the biosynthetic enzyme for LTB4, in patients with leg LE.

\section{Systemic Inflammatory Response}

Interestingly, one study using near-infrared fluorescent imaging (NIRFLI) found lymphatic architecture abnormalities in both the affected and normal arm in patients with unilateral $\mathrm{BCRL}$, and the percentage of aberrant lymphatics increased 
with time after initial onset of LE, suggesting that the inflammatory response in LE is systemic. ${ }^{35-39}$ Supporting this finding, in a murine model of acute inflammation, systemic lymph propulsive frequency (pumping) and velocity measured using NIRFLI dramatically decreased after a lipopolysaccharide (LPS)-mediated inflammatory insult, before gradually returning to normal values after several days. Changes were not only local, ${ }^{40-42}$ but systemic (i.e., occurring on both sides of the mouse), despite inflammatory insult on only one side. ${ }^{43}$ IL- $1 \beta$, TNF- $\alpha$, and IL- 6 cytokines were found to be elevated in serum during lymphatic pumping cessation and were found to systemically decrease lymphatic pulsing frequency and velocity following intradermal administration, and this process was ameliorated by pre-treatment with an iNOS inhibitor, suggesting a nitric oxide-mediated mechanism. ${ }^{44}$ These results suggest that systemic anti-inflammatory treatments may be beneficial in the treatment of LE.

\section{Mesenchymal Stem Cell Therapy}

Studies have demonstrated that mesenchymal stem cells can differentiate into lymphatic endothelial-like cells in specialized in vitro culture conditions and can increase drainage of interstitial fluid when injected in vivo. ${ }^{45}$ For this reason, bone marrow- (BM-MSCs) or adipose tissue-derived mesenchymal stem cells (AT-MSCs) may provide a therapeutic benefit in patients with LE.

\section{Bone Marrow-derived Mesenchymal Stem Cells}

BM-MSCs have been investigated for the treatment of LE. In one study, patients with upper extremity BCRL who underwent intramuscular and subcutaneous injection with autologous BM-MSCs into the axillary region and upper arm were compared with controls who underwent complex decongestive therapy (CDT). ${ }^{46}$ The investigators found that the volume reduction was higher and pain scores were lower in patients treated with BM-MSCs compared with the control group during a 12-month follow-up. A similar study found no significant differences in volume reduction between the autologous BMMSC and control group treated with CDT at 12 weeks follow-up; however, pain and sensitivity were reduced. ${ }^{47}$

\section{Adipose Tissue-Derived Mesenchymal Stem Cells}

Recent studies have suggested that the use of AT-MSCs may have benefit in patients with LE. In a small pilot study using AT-MSCs injected directly into the axillary region and combined with a scar-releasing fat graft procedure in patients with BCRL with 6 months follow-up, a small volume reduction was noted, but was not significant. Half of the patients, however, reduced their use of conservative management, and patient-reported outcomes improved significantly over time. ${ }^{48}$ In another study, BCRL patients with pitting edema of the hand and forearm underwent lipofilling under the axillary vein. Limb volume was significantly reduced in the majority of patients within a 24-month follow-up, and symptoms, including arm heaviness, numbness, and functional impairment of the upper limb in daily activities, were reduced. ${ }^{49}$ In an interesting study using a murine hindlimb model of LE, only combination treatment with vascularized lymph node transfer with adipose-derived stem cells demonstrated a significant reduction in hind paw volume, ${ }^{50}$ suggesting possible clinical benefit in augmenting the outcomes of vascularized lymph node transfer.

\section{VEGF-C Therapy}

Vascular endothelial growth factor- $\mathrm{C}$ is a critical regulator of lymphangiogenesis, and through VEGF receptor 2 and 3 signaling regulates a wide range of effects in LECs, including differentiation, survival, and migration. Experimental LE models using exogenous delivery of VEGF-C at supraphysiological doses or gene therapy delivery with adenoviral vectors have demonstrated lymphangiogenesis and decreased edema in the treatment groups. ${ }^{51,52}$ Using a murine model, the therapeutic effects of different VEGFs, in combination with lymph node transfer, were examined at 3 months postoperatively. The VEGF-C group demonstrated the greatest lymphangiogenic response of all of the growth factors. ${ }^{53}$ In another study examining axillary wound exudate samples in patients following lymph node transfer, the levels of the anti-inflammatory and anti-fibrotic cytokine IL-10, as well as VEGF-C, were increased. ${ }^{54}$ Of note, expression of VEGF-C is markedly increased in lymphedematous tissues, suggesting that formation of collateral lymphatics in this scenario is actively inhibited, ${ }^{27}$ possibly by $\mathrm{T}$ cell-derived cytokines decreasing the responsiveness of LECs to lymphangiogenic growth factors. ${ }^{15}$ In addition, in a porcine LE model using aligned nanofibrillar collagen scaffolds positioned across the area of lymphatic obstruction, coating the scaffold with VEGF-C impeded the functional therapeutic response, with the development of nonfunctional vascular proliferation in the presence of VEGF-C-coated threads.

Clinical translation of VEGF-C (or any other lymphangiogenic growth factors) therapy in cancer survivors, however, has been limited by the concern of potential to increase tumor metastasis and malignant behavior in a variety of solid tumors by regulating infiltration and migration of tumorassociated macrophages, in addition to other effects on the tumor microenvironment. ${ }^{55,56} \mathrm{~A}$ phase I multicenter clinical study in patients with BCRL is currently underway to assess the safety and efficacy of using a VEGF-C adenoviral vector in combination with vascularized lymph node transfer. Another pilot study took a different approach, using a VEGF-C inhibitor in patients with BCRL, and found that interstitial fluid pressure and extracellular fluid volume were reduced following treatment. ${ }^{57}$ Although the mean difference in excess arm volume did not significantly change, half of the patients met the endpoint of $a \geq 25 \%$ reduction in excess arm volume 4 weeks after initiating treatment. Patients, however, experienced a greater-than-expected toxicity at the dose used, and further studies using lower doses are required.

\section{Fabricated Scaffolds}

Mechanically crossing the region of the obstruction of lymphatic flow by lymphangioplasty has long been of interest as 
a treatment for LE. ${ }^{58-60}$ Using aligned nanofibrillar collagen scaffolds positioned across the area of lymphatic obstruction, in a porcine model of acquired LE, a study compared outcomes using the scaffolds either alone, in conjunction with autologous lymph node transfer, or supplemented with exogenous VEGF-C, with a control group. ${ }^{61}$ At 3 months post implantation, immunofluorescence staining demonstrated a significant increase in lymphatic collectors within close proximity to the scaffolds, and bioimpedance spectroscopy demonstrated a significant reduction in extracellular fluid, as well as quantifiable lymphatic collectors visualized by contrast-enhanced computed tomography, in the groups with the scaffolds, and groups with scaffolds and lymph node transfer. Following the encouraging results of this study, a clinical trial of using the scaffold in conjunction with vascularized lymph node transfer surgery is currently underway. Tissue-engineered solutions for LE, therefore, represent a very interesting future direction for LE treatment. ${ }^{61-65}$

\section{Conclusion}

Exciting advances in our understanding of the pathophysiology underlying LE have led to several new and emerging therapies that hold promise for the treatment of LE. Further understanding of the genetics and the complex interplay with secondary mechanisms will lead to further targeted therapeutic options to alleviate suffering in patients with this prevalent disease for which there is currently no cure.

\section{Conflict of Interest}

None.

\section{References}

1 Shaitelman SF, Chiang YJ, Griffin KD, et al. Radiation therapy targets and the risk of breast cancer-related lymphedema: a systematic review and network meta-analysis. Breast Cancer Res Treat 2017;162(02):201-215

2 Rutkowski JM, Swartz MA. A driving force for change: interstitial flow as a morphoregulator. Trends Cell Biol 2007;17(01):44-50

3 Mihara M, Hara H, Hayashi Y, et al. Pathological steps of cancerrelated lymphedema: histological changes in the collecting lymphatic vessels after lymphadenectomy. PLoS One 2012;7(07):e41126

4 Olszewski WL, Jamal S, Manokaran G, Lukomska B, Kubicka U. Skin changes in filarial and non-filarial lymphoedema of the lower extremities. Trop Med Parasitol 1993;44(01):40-44

5 Zampell JC, Yan A, Elhadad S, Avraham T, Weitman E, Mehrara BJ. $\mathrm{CD} 4(+)$ cells regulate fibrosis and lymphangiogenesis in response to lymphatic fluid stasis. PLoS One 2012;7(11):e49940

6 Suami H, Pan WR, Taylor GI. Changes in the lymph structure of the upper limb after axillary dissection: radiographic and anatomical study in a human cadaver. Plast Reconstr Surg 2007;120(04):982-991

7 Avraham T, Daluvoy S, Zampell J, et al. Blockade of transforming growth factor-beta1 accelerates lymphatic regeneration during wound repair. Am J Pathol 2010;177(06):3202-3214

8 Ly CL, Kataru RP, Mehrara BJ. Inflammatory Manifestations of lymphedema. Int J Mol Sci 2017;18(01):E171

9 Kataru RP, Kim H, Jang C, et al. T lymphocytes negatively regulate lymph node lymphatic vessel formation. Immunity 2011;34(01): 96-107

10 Zampell JC, Avraham T, Yoder N, et al. Lymphatic function is regulated by a coordinated expression of lymphangiogenic and anti-lymphangiogenic cytokines. Am J Physiol Cell Physiol 2012; 302(02):C392-C404

11 Wynn TA. Cellular and molecular mechanisms of fibrosis. J Pathol 2008;214(02):199-210

12 Gousopoulos E, Proulx ST, Scholl J, Uecker M, Detmar M. Prominent lymphatic vessel hyperplasia with progressive dysfunction and distinct immune cell infiltration in lymphedema. Am J Pathol 2016;186(08):2193-2203

13 Gardenier JC, Kataru RP, Hespe GE, et al. Topical tacrolimus for the treatment of secondary lymphedema. Nat Commun 2017; 8:14345

14 Avraham T, Zampell JC, Yan A, et al. Th2 differentiation is necessary for soft tissue fibrosis and lymphatic dysfunction resulting from lymphedema. FASEB J 2013;27(03):1114-1126

15 Savetsky IL, Ghanta S, Gardenier JC, et al. Th2 cytokines inhibit lymphangiogenesis. PLoS One 2015;10(06):e0126908

16 Shin K, Kataru RP, Park HJ, et al. TH2 cells and their cytokines regulate formation and function of lymphatic vessels. Nat Commun 2015;6:6196

17 Gousopoulos E, Proulx ST, Bachmann SB, et al. Regulatory T cell transfer ameliorates lymphedema and promotes lymphatic vessel function. JCI Insight 2016;1(16):e89081

18 García Nores GD, Ly CL, Savetsky IL, et al. T-regulatory cells mediate local immunosuppression in lymphedema. J Invest Dermatol 2017:S0022-202X(17)32961-5

19 Clavin NW, Avraham T, Fernandez J, et al. TGF-beta1 is a negative regulator of lymphatic regeneration during wound repair. Am J Physiol Heart Circ Physiol 2008;295(05):H2113-H2127

20 Gardenier JC, Hespe GE, Kataru RP, et al. Diphtheria toxinmediated ablation of lymphatic endothelial cells results in progressive lymphedema. JCI Insight 2016;1(15):e84095

21 Harvey NL, Srinivasan RS, Dillard ME, et al. Lymphatic vascular defects promoted by Prox1 haploinsufficiency cause adult-onset obesity. Nat Genet 2005;37(10):1072-1081

22 Cuzzone DA, Weitman ES, Albano NJ, et al. IL-6 regulates adipose deposition and homeostasis in lymphedema. Am J Physiol Heart Circ Physiol 2014;306(10):H1426-H1434

23 Scallan JP, Zawieja SD, Castorena-Gonzalez JA, Davis MJ. Lymphatic pumping: mechanics, mechanisms and malfunction. J Physiol 2016;594(20):5749-5768

24 Torrisi JS, Hespe GE, Cuzzone DA, et al. Inhibition of inflammation and iNOS improves lymphatic function in obesity. Sci Rep 2016; 6:19817

25 Cintolesi V, Stanton AW, Bains SK, et al. Constitutively enhanced lymphatic pumping in the upper limbs of women who later develop breast cancer-related lymphedema. Lymphat Res Biol 2016;14(02):50-61

26 Liao S, Cheng G, Conner DA, et al. Impaired lymphatic contraction associated with immunosuppression. Proc Natl Acad Sci U S A 2011;108(46):18784-18789

27 Rutkowski JM, Moya M, Johannes J, Goldman J, Swartz MA. Secondary lymphedema in the mouse tail: Lymphatic hyperplasia, VEGF-C upregulation, and the protective role of MMP-9. Microvasc Res 2006;72(03):161-171

28 Ghanta S, Cuzzone DA, Torrisi JS, et al. Regulation of inflammation and fibrosis by macrophages in lymphedema. Am J Physiol Heart Circ Physiol 2015;308(09):H1065-H1077

29 Ogata F, Fujiu K, Matsumoto S, et al. Excess lymphangiogenesis cooperatively induced by macrophages and CD4(+) T cells drives the pathogenesis of lymphedema. J Invest Dermatol 2016;136 (03):706-714

30 Zampell JC, Elhadad S, Avraham T, et al. Toll-like receptor deficiency worsens inflammation and lymphedema after lymphatic injury. Am J Physiol Cell Physiol 2012;302(04): C709-C719

31 Tian W, Rockson SG, Jiang X, et al. Leukotriene B4 antagonism ameliorates experimental lymphedema. Sci Transl Med 2017;9 (389):3920 
32 Peters-Golden M, Henderson WR Jr. Leukotrienes. N Engl J Med 2007;357(18):1841-1854

33 Nakamura K, Radhakrishnan K, Wong YM, Rockson SG. Antiinflammatory pharmacotherapy with ketoprofen ameliorates experimental lymphatic vascular insufficiency in mice. PLoS One 2009;4(12):e8380

34 Jiang X, Nicolls MR, Tian W, Rockson SG. Lymphatic dysfunction, leukotrienes, and lymphedema. Annu Rev Physiol 2017

35 Aldrich MB, Guilliod R, Fife CE, et al. Lymphatic abnormalities in the normal contralateral arms of subjects with breast cancerrelated lymphedema as assessed by near-infrared fluorescent imaging. Biomed Opt Express 2012;3(06):1256-1265

36 Bains SK, Peters AM, Zammit C, et al. Global abnormalities in lymphatic function following systemic therapy in patients with breast cancer. Br J Surg 2015;102(05):534-540

37 Burnand KM, Glass DM, Mortimer PS, Peters AM. Lymphatic dysfunction in the apparently clinically normal contralateral limbs of patients with unilateral lower limb swelling. Clin Nucl Med 2012;37(01):9-13

38 Pain SJ, Barber RW, Ballinger JR, et al. Local vascular access of radioprotein injected subcutaneously in healthy subjects and patients with breast cancer-related lymphedema. J Nucl Med 2004;45(05):789-796

39 Pain SJ, Purushotham AD, Barber RW, et al. Variation in lymphatic function may predispose to development of breast cancer-related lymphoedema. Eur J Surg Oncol 2004;30(05):508-514

40 Hanley CA, Elias RM, Movat HZ, Johnston MG. Suppression of fluid pumping in isolated bovine mesenteric lymphatics by interleukin-1: interaction with prostaglandin E2. Microvasc Res 1989;37 (02):218-229

41 Wee JL, Greenwood DL, Han X, Scheerlinck JP. Inflammatory cytokines IL-6 and TNF- $\alpha$ regulate lymphocyte trafficking through the local lymph node. Vet Immunol Immunopathol 2011;144(1-2):95-103

42 Baluk P, Yao LC, Feng J, et al. TNF-alpha drives remodeling of blood vessels and lymphatics in sustained airway inflammation in mice. J Clin Invest 2009;119(10):2954-2964

43 Aldrich MB, Sevick-Muraca EM. Cytokines are systemic effectors of lymphatic function in acute inflammation. Cytokine 2013;64 (01):362-369

44 Chen Y, Rehal S, Roizes S, Zhu HL, Cole WC, von der Weid PY. The pro-inflammatory cytokine TNF-alpha inhibits lymphatic pumping via activation of the NF-kB-iNOS signaling pathway. Microcirculation 2017. Doi: 10.1111/micc.12364

45 Conrad C, Niess H, Huss R, et al. Multipotent mesenchymal stem cells acquire a lymphendothelial phenotype and enhance lymphatic regeneration in vivo. Circulation 2009;119(02):281-289

46 Hou $\mathrm{C}, \mathrm{Wu} \mathrm{X}$, Jin X. Autologous bone marrow stromal cells transplantation for the treatment of secondary arm lymphedema: a prospective controlled study in patients with breast cancer related lymphedema. Jpn J Clin Oncol 2008;38(10):670-674

47 Maldonado GE, Pérez CA, Covarrubias EE, et al. Autologous stem cells for the treatment of post-mastectomy lymphedema: a pilot study. Cytotherapy 2011;13(10):1249-1255

48 Toyserkani NM, Jensen CH, Andersen DC, Sheikh SP, Sørensen JA. Treatment of breast cancer-related lymphedema with adipose- derived regenerative cells and fat grafts: a feasibility and safety study. Stem Cells Transl Med 2017;6(08):1666-1672

49 Vandermeeren L, Belgrado JP, Vankerckhove S, et al. Lipofilling of the axilla to reduce secondary lymphedema after axillary lymph node dissection. Eur J Cancer 2016;57(Suppl 2):72

50 Hayashida K, Yoshida S, Yoshimoto H, et al. Adipose-derived stem cells and vascularized lymph node transfers successfully treat mouse hindlimb secondary lymphedema by early reconnection of the lymphatic system and lymphangiogenesis. Plast Reconstr Surg 2017;139(03):639-651

51 Baker A, Kim H, Semple JL, et al. Experimental assessment of prolymphangiogenic growth factors in the treatment of post-surgical lymphedema following lymphadenectomy. Breast Cancer Res 2010;12(05):R70

52 Hartiala P, Saarikko AM. Lymphangiogenesis and lymphangiogenic growth factors. J Reconstr Microsurg 2016;32(01):10-15

53 Tervala TV, Hartiala P, Tammela T, et al. Growth factor therapy and lymph node graft for lymphedema. J Surg Res 2015;196(01): 200-207

54 Viitanen TP, Visuri MT, Sulo E, Saarikko AM, Hartiala P. Antiinflammatory effects of flap and lymph node transfer. J Surg Res 2015;199(02):718-725

55 Skobe M, Hawighorst T, Jackson DG, et al. Induction of tumor lymphangiogenesis by VEGF-C promotes breast cancer metastasis. Nat Med 2001;7(02):192-198

56 Shiao SL, Ganesan AP, Rugo HS, Coussens LM. Immune microenvironments in solid tumors: new targets for therapy. Genes Dev 2011;25(24):2559-2572

57 Miller KD, Brown C, Perkins SM, Schneider BP, Storniolo A, Sledge GW. A pilot study of vascular endothelial growth factor inhibition with pazopanib in patients (pts) with lymphedema following breast cancer treatment. Cancer Res 2010;70(Suppl 24):P2-14-02

58 Handley WS. Two Cases of Lymphangioplasty for the brawny arm of breast cancer. Proc R Soc Med 1908;1(Clin Sect):186-190

59 Silver D, Puckett CL. Lymphangioplasty: a ten year evaluation. Surgery 1976;80(06):748-755

60 Olszewski WL, Zaleska M. Treatment of postmastectomy lymphedema by bypassing the armpit with implanted silicone tubings. Int Angiol 2017;36(01):50-58

61 Hadamitzky C, Zaitseva TS, Bazalova-Carter M, et al. Aligned nanofibrillar collagen scaffolds - guiding lymphangiogenesis for treatment of acquired lymphedema. Biomaterials 2016;102:259-267

62 Kanapathy M, Patel NM, Kalaskar DM, Mosahebi A, Mehrara BJ, Seifalian AM. Tissue-engineered lymphatic graft for the treatment of lymphedema. J Surg Res 2014;192(02):544-554

63 Kanapathy M, Kalaskar D, Mosahebi A, Seifalian AM. Development of a tissue-engineered lymphatic graft using nanocomposite polymer for the treatment of secondary lymphedema. Artif Organs 2016;40(03):E1-E11

64 Huang NF, Okogbaa J, Lee JC, et al. The modulation of endothelial cell morphology, function, and survival using anisotropic nanofibrillar collagen scaffolds. Biomaterials 2013;34(16):4038-4047

65 Nakayama KH, Hong G, Lee JC, et al. Aligned-braided nanofibrillar scaffold with endothelial cells enhances arteriogenesis. ACS Nano 2015;9(07):6900-6908 